\title{
Factors that influence users' adoption of being coached by an Artificial Intelligence Coach
}

\author{
Nicky Terblanche \\ University of Stellenbosch Business School \\ University of Stellenbosch \\ Cape Town, South Africa \\ Danie Cilliers \\ University of Stellenbosch Business School \\ University of Stellenbosch \\ Cape Town, South Africa
}

\begin{abstract}
The rise of artificial intelligence (AI) is making in-roads into many spheres of life, including workplace coaching. The introduction of a new class of support technologies ('e-coaching systems' or 'AI Coaching') promise to deliver personalised, timely, around-the-clock coaching in a wide variety of domains and to a broad audience. Chatbots as a type of e-coaching system and a form of Weak $\mathrm{AI}$ in particular, has the potential to replace or augment human coaches in certain instances, however it seems that speculation and hype is clouding our understanding of its true potential. This is reminiscent of the lack of evidence-based practice in coaching itself. To prevent AI Coaching from following a similar route, empirical research is needed. In this paper we summarise the findings of one of the first ever studies on the use of AI in organisational coaching. We used the Unified Theory of Acceptance and Use of Technology (UTAUT) as a theoretical framework to examine the determinants associated with individuals' behavioural intention to use an AI Coach (a goal-attainment chatbot called Vicci). A total of 226 users had a coaching conversation with Vicci and then completed the UTAUT survey. Determinants of behavioural intention were measured: performance and effort expectancies, social influence, facilitating conditions, attitude and perceived risk. Structural equation modelling analysis revealed that performance expectancy, social influence and attitude are the main determinants of behavioural intent, while age, gender and level of goal attainment play a moderating role.
\end{abstract}

Keywords: coaching; workplace coaching; artificial intelligence; chatbots; conversational agents; technology acceptance; UTAUT

This is an Open Access article distributed under the terms of the Creative Commons Attribution (CC BY) License which permits use, distribution and reproduction in any medium, provided the original work is properly cited. 


\section{Introduction}

The Fourth Industrial Revolution at the World Economic Forum in Davos in 2016 (Schwab, 2017) has contributed to a renewed interest in artificial intelligence (AI). Since then much has been speculated about the potential role AI could play in society (Acemoglu \& Restrepo, 2018; Brynjolfsson \& McAfee, 2012). The application of AI currently spans numerous industries, including the helping professions such as healthcare, psychology and coaching (Pereira \& Diaz, 2019). While the use of AI in healthcare has received significant attention from a research perspective (see for example Boh et al., 2016; Kamphorst, 2017), research in the coaching domain is sparse. This is somewhat surprising since coaching is considered one of the fastest growing professions in the world (Theeboom, Beersma, \& Vianen, 2013). Given that AI is likely to continue its upward trajectory, it is important to conduct empirical research on the application of $\mathrm{AI}$ in coaching in order to understand its potential and limitations, based on evidence. As a first foray into the study of AI and coaching, we created an AI Coach using chatbot technology and goalattainment theory. The chatbot was used in an empirical study that sought an answer to the question, what are the factors that influence the users' adoption of being coached by an AI Coach? This paper summaries the findings from that exploratory study.

\section{Coaching and AI}

In this paper 'coaching' refers to a one-on-one structured conversation between a coach and client with the aim of facilitating sustainable change for the individual and potentially other stakeholders (Bachkirova, Cox, \& Clutterbuck, 2014). Traditional coaching involves a human coach and human client interacting with each other face-to-face. However, this traditional mode of interaction is changing. The $12^{\text {th }}$ Sherpa Coaching Survey (2017) showed that the percentage of coaching delivered face-to-face decreased in the last seven years and accounts for only approximately $32 \%$ of all coaching interactions. BetterUp (Lopez, 2018) and Sharpist (Weimer, 2019) are examples of new technology platforms that have been launched recently that offer services like coach matching, administration of coaching sessions and ecoaching. In e-coaching a coach uses various modalities such as asynchronous text based (e.g. email), synchronous text based (e.g. WhatsApp), audio (e.g. telephone) and video (e.g. Skype) to communicate with a client (Geissler, Hasenbein, Kanatouri, \& Wegener, 2014). As such, the use of technology in coaching is not novel, although the application of AI in coaching is relatively new and thus the focus of this investigation. 
AI is defined as "the broad collection of technologies, such as computer vision, language processing, robotics, robotic process automation and virtual agents that are able to mimic cognitive human functions" (Bughin \& Hazan, 2017 , p. 4). A distinction can be made between artificial narrow intelligence or Weak AI (systems that can perform only a very specific task in a narrow context), and artificial general intelligence or Strong AI (systems that are at least as intelligent as humans and can apply their learning in different contexts) (Siau \& Yang, 2017). Strong AI is at this stage in its infancy with no clear indication of reaching maturity in the foreseeable future. Weak AI is showing steady signs of progress with encouraging results in specific applications such as speech recognition and self-driving cars (Panetta, 2018).

For the purpose of this investigation, a form of Weak AI was implemented to simulate a human coach. Specifically, a chatbot (conversational agent) named Vicci was created to assist users with goal attainment. Chatbots are a category of software agents or computer programs that engage in human conversation through either auditory, textual or mixed methods (Hatwar, Patil, \& Gondane, 2016). Vicci employs goal-attainment theory (Grant, 2011) and the GROW model (Grant, 2011) to facilitate a conversation with a human in regards to setting realistic goals and action plans, and following up on progress.

As a novel application of technology in coaching, two aspects of AI Coaching are currently not well understood. First, what are the factors that influence the uptake of this new technology? Second, how effective is it? In this study, the question of uptake was investigated through the application of the Unified Theory of Acceptance and Use of Technology (UTAUT) model.

\section{Technology acceptance models}

In information systems, the study of factors that influence the acceptance and rejection of new technologies is often undertaken using various incarnations of the technology acceptance model (TAM) (Venkatesh, Morris, Davis \& Davis, 2003).

To investigate the uptake of AI in coaching, we employed the UTAUT, a version of TAM often used in product or service-oriented technology research (Venkatesh et al., 2003). The augmented UTAUT models the factors that influence the behavioural intent (BI) of a user to use an AI chatbot coach. It consists of the six constructs summarised in Table 1. 
Table 1. UTAUT Construct definitions

\begin{tabular}{ll}
\hline Construct & Definition \\
\hline Performance expectancy & $\begin{array}{l}\text { "The degree to which an individual believes that the chatbot will } \\
\text { help him or her to attain gains in performance" (Venkatesh et al., } \\
\text { 2003, p.447). }\end{array}$ \\
\hline Effort expectancy & "The degree of ease associated with the use of the chatbot" \\
(Venkatesh et al., 2003, p. 450). \\
social influence \\
$\begin{array}{l}\text { "The degree to which an individual perceived that important others } \\
\text { believe he or she should use the chatbot" (Venkatesh et al., 2003, p. } \\
\text { 451). }\end{array}$ \\
$\begin{array}{l}\text { "The degree to which an individual believes that an organisational } \\
\text { and technical infrastructure exists to support the use of the chatbot" } \\
\text { (Venkatesh et al., 2003, p. 456). }\end{array}$ \\
\hline $\begin{array}{l}\text { The degree to which the user of chatbot coaching believes that s/he } \\
\text { may be exposed to certain types of risks (adapted from Zhang, Zhu, } \\
\text { \& Liu, 2012). Includes breaches of confidentiality, trust issues, and } \\
\text { perceptions that AI is a threat towards humans. }\end{array}$ \\
\hline $\begin{array}{l}\text { An individual's positive or negative feelings about performing the } \\
\text { target behaviour. Venkatesh et al. (2003) describe it as an } \\
\text { individual's overall affective reaction to using a system, in this case } \\
\text { the coach chatbot. }\end{array}$ \\
$\begin{array}{l}\text { A measure of a person's perceived likelihood or relative strength of } \\
\text { intention to perform a particular behaviour, such as the use of a } \\
\text { chatbot. }\end{array}$ \\
\hline
\end{tabular}

\section{Research Design}

The Vicci coaching chatbot was made available on an instant messaging platform and participants (sourced online via snowball sampling) were invited to have at least one conversation with the chatbot before completing an online UTAUT survey. The validated UTAUT survey consisted of questions that relate to the constructs in Table 1. A total of 226 valid responses were received. Analysis was performed using partial least square structural equation modelling (PLS-SEM). The direct effects of all the constructs in Table 1 on BI were measured, as well as the moderating effect of age, gender and level of goal 
attainment. As this is a summarised version of the study, a detailed description of the data analysis falls outside the scope of this paper.

\section{Results and Discussion}

The first of two major findings relate to the direct impact of the UTAUT factors on BI. Of the six factors investigated, three were found to be statistically significant determinants of BI. The first of these, Performance Expectancy (PE) suggests that users valued what they gained from using the chatbot coach. Next, Social Influence (SI) indicates that users appear to care about what other people (whom they know) think about the chatbot. Finally, Attitude (AT) was also found to be important; the positive or negative feelings a user has about using the chatbot coach. This first set of findings suggests that users will be more willing to use Vicci when they feel a positive attitude towards the chatbot and the coaching experience, when they get tangible value from the coaching intervention, and when their friends, family and co-workers are also using the chatbot for coaching. These findings confirm the value-seeking and engaging relationship character requirement for chatbots within a coaching context (Cavanagh \& Grant, 2018; Grant, 2011, 2012).

In contrast, the other three factors - effort expectancy (EE), facilitating conditions (FC), and perceived risks (PR) - showed no significant effect on BI. For these factors, EE refers to the ease associated with using the chatbot coach; FC refers to the availability of the necessary technical infrastructure needed to use the chatbot, whilst PR is users' perceived the level of risk exposure from using the technology. Since these factors had no statistically significant impact on $\mathrm{BI}$, the conclusion is drawn that over-investing in infrastructure support and over-simplification of Vicci will not necessarily result in higher user acceptance. Of the three statistically significant factors, performance expectancy had the strongest impact implying that Vicci users are likely to accept chatbots primarily for the potential they have to enhance performance, and less so for reasons related to ease of use, technical support and/or risk perceptions.

The second major finding relates to the moderating effects of age, gender and goal attainment on BI. Age moderated the effect of SI on BI, with older people more influenced by friends, family and colleagues' perceptions of the chatbot coach. Gender moderated the role of PR in BI, with males more influenced by the perceived risks associated with using the chatbot coach than females. Finally, when users felt that the chatbot helped them to achieve their goals, the role of SI on BI decreased. 


\section{Implications for practice}

These exploratory findings indicate that performance expectations play a central role in an individual's intention to use a coaching chatbot. As such, chatbot coach designers should collaborate with coaching researchers and practitioners to develop chatbots that have a high perceived usefulness. This can be achieved by using robust evidence-based coaching frameworks in the chatbot design. Designers must ensure that the chosen theoretical models have been empirically validated in the organisational coaching context to avoid the current pitfall of using untested coaching models and frameworks in practice (Spence \& Oades, 2011). In addition, as social influence seems to play a role in the uptake of the chatbot coach, organisations wishing to deploy a chatbot coaching should consider using influencers who are early-adopters and have positive attitudes towards the technology, as they can act as internal advocates for the use of chatbots. Practically, a significant benefit of a coaching chatbot is that it is available at all times, and can handle several conversations at the same time. The opportunity to scale coaching cost-effectively to larger audiences where a coach was previously not accessible, is a further implication for organisations.

\section{Implications for coaching research}

Coaching as an emerging profession has suffered in reputation from unscientific praxis and unsubstantiated claims of efficacy (Grant, 2014). The rise of AI in coaching appears to follow a similar trajectory with numerous speculative opinions in the popular press about how AI might impact coaching. The research presented here is the first in a series of projects undertaken by the first author to rigorously and empirically examine the use of AI in coaching, with the aim of providing evidence-based recommendations. From the present study we tentatively conclude that what "works" in human-to-human coaching may not necessarily be applicable in AI-to-human coaching. In traditional coaching, the coaching relationship is considered one of the most important determinants of coaching success (De Haan, Culpin, \& Curd, 2011) with trust being a key ingredient of a strong relationship (Grant, 2014). In AI Coaching one could therefore speculate that the trust relationship between the user and AI Coach must also be important, but that users of a chatbot coach may struggle to trust an algorithm (non-human), which could therefore lead to a weak relationship and negative coaching outcomes.

However, the findings of this study suggest that trust may not be as important in usage uptake as the perceived usefulness of the chatbot. It may be 
that users are prepared to overlook potential risks when the chatbot is perceived to help them achieve their goals. Such a finding is important and worthy of further investigation. Blindly applying what we know about human-to-human coaching to the AI-to-human coaching context may lead to incorrect assumption and suboptimal AI Coaches. We should only draw conclusions about AI Coaching based on empirical research.

The present study looked at technology acceptance. Research is also needed into the efficacy of AI Coaching. To this end a longitudinal, randomised control trial study is currently underway to compare the goal attainment of participants who used Vicci for eight months relative to a no-coaching control group. This study will be compared to a similar one involving human coaches. In the future, a hybrid approach where participants are exposed to human coaches who use Vicci as a coaching aid will be conducted to investigate the potential of AI Coaching as an augmentation to and not a replacement of human coaching.

\section{Conclusion}

At a time where much is speculated about the potential of AI, it is important to gather evidence of AI's applicability to coaching, not least to ensure AI coaching can be implemented in a responsible and considered manner. This study represents an initial step towards building knowledge in this important area. The suggestions provided here could be of benefit to the purchasers, designers and users of chatbot coaching and will hopefully lead to more active participant from the coaching community in this emerging and potentially powerful coaching capability.

\section{References}

Acemoglu, D., \& Restrepo, P. (2018). The race between man and machine: Implications of technology for growth, factor shares, and employment. American Economic Review, 108(6), 1488-1542.

Bachkirova, T., Cox, E., \& Clutterbuck, D. (2014). Introduction. In E. Cox, T. Bachkirova \& D. Clutterbuck (eds.), The Complete Handbook of Coaching. Sage (pp. 1-20). 
Boh, B., Lemmens, L. H. J. M., Jansen, A., Nederkoorn, C., Kerkhofs, V., Spanakis, G., Roefs, A. (2016). An ecological momentary intervention for weight loss and healthy eating via smartphone and internet: Study protocol for a randomised controlled trial. Trials, 17(1), 154.

Brynjolfsson, E., \& McAfee, A. (2012). Race against the machine: How the digital revolution is accelerating innovation, driving productivity, and irreversibly transforming employment and the economy. MIT Center for Digital Business research brief. Retrieved from https://doi.org/10.1016/S2213-8587(14)70016-6

Bughin, J., \& Hazan, E. (2017). The new spring of artificial intelligence: A few early economies. Retrieved from https://voxeu.org/article/new-springartificial-intelligence-few-early-economics

Cavanagh, M., \& Grant, A. (2018). The solution-focused approach to coaching. In E. Cox, T. Bachkirova \& D. Clutterbuck (eds.), The Complete Handbook of Coaching. Sage (pp. 51-64).

De Haan, E., Culpin, V., \& Curd, J. (2011). Executive coaching in practice: What determines helpfulness for clients of coaching? Personnel Review, 40(1), 24-44.

Geissler, H., Hasenbein, M., Kanatouri, S., \& Wegener, R. (2014). E-Coaching: Conceptual and empirical findings of a virtual coaching programme. International Journal of Evidence Based Coaching and Mentoring, 12(2), 165-186.

Grant, A. M. (2011). Is it time to REGROW the GROW model? Issues related to teaching coaching session structures. The Coaching Psychologist, $7(2), 85-172$.

Grant, A. M. (2012). An integrated model of goal-focused coaching: An evidence-based framework for teaching and practice. International Coaching Psychology Review, 7(2), 146-165.

Grant, A.M. (2014). Autonomy support, relationship satisfaction and goal focus in the coach-coachee relationship: Which best predicts coaching success? Coaching: An International Journal of Theory, Research and Practice, 7(1), 18-38. 
Hatwar, N., Patil, A., \& Gondane, D. (2016). AI based chatbot. International Journal of Emerging Trends in Engineering and Basic Sciences, 3(2), $85-87$.

Kamphorst, B. A. (2017). E-coaching systems: What they are, and what they aren't. Personal and Ubiquitous Computing, 21(4), 625-632.

Lopez, M. (2018). BetterUp Raises \$26 Million To Democratize And Enhance Coaching With AI And Mobility. Forbes. Com. Retrieved from https://www.forbes.com/sites/maribellopez/2018/03/28/betterup-raises26-million-to-democratize-and-enhance-coaching-with-ai-andmobility/\#2141bd2ef496

Panetta, K. (2018). Widespread artificial intelligence, biohacking, new platforms and immersive experiences dominate this year's Gartner Hype Cycle. Retrieved from https://www.gartner.com/smarterwithgartner/5trends-emerge-in-gartner-hype-cycle-for-emerging-technologies-2018/

Pereira, J., \& Diaz, O. (2019). Using Health Chatbots for Behavior Change: A Mapping Study. Journal of Medical Systems, 43(5), 135.

Schwab, K. (2017). The Fourth Industrial Revolution: What it Means and How to Respond. Crown Publishing Group.

Sherpa. (2017). Executive Coaching Survey: The 12th annual industry review from sherpa coaching. Retrieved June 16, 2019, from http://www.sherpacoaching.com/pdf files/2017_Executive_Coaching_Survey_FINAL.pdf

Siau, KL. \& Yang, Y. (2017). Impact of Artificial Intelligence, Robotics, and Machine Learning on Sales and Marketing. Midwest United States for Information Systems Conference Proceedings (MWAIS 2017), 48. Retrieved from http://aisel.aisnet.org/mwais2017/48

Spence, G. B., \& Oades, L. G. (2011). Coaching with self-determination theory in mind: Using theory to advance evidence-based coaching practice. International Journal of Evidence-Based Coaching and Mentoring, 9(2), $37-55$. 
Theeboom, T., Beersma, B., \& Van Vianen, A. (2013). Does coaching work? A meta-analysis on the effects of coaching on individual level outcomes in an organizational context. The Journal of Positive Psychology, 9(1), 118.

Venkatesh, V., Morris, M. G., Davis, G. B., \& Davis, F. D. (2003). User acceptance of information technology: Toward a unified view. MIS Quarterly, 27(3), 425-478.

Weimer, M. (2019). Millionen für den Online-Coach für Führungskräfte. Retrieved January 10, 2020, from

https://www.gruenderszene.de/karriere/online-coach-fuehrungskraeftesharpist?interstitial_click

Zhang, L., Zhu, J., \& Liu, Q. (2012). A meta-analysis of mobile commerce adoption and the moderating effect of culture. Computers in Human Behavior, 28(5), 1902-1911.

\section{Author Contact}

Dr Nicky Terblanche

University of Stellenbosch Business School

University of Stellenbosch

Stellenbosch

South Africa

E: nickyt@usb.ac.za 